\title{
Reviewing Translation: Putting Houses In Order
}

\begin{abstract}
This paper attempts to address, as exhaustively as possible, all the questions pertaining to the act of reviewing translations. For most it provides detailed likely answers, including both sides of each issue (the translator's and the reviewer's). In addition it attempts to identify some of the major areas in which (a) translators may have to alter their attitude or work or both so that reviewers can (will be forced to) do their job better, and (b) publishers of books, magazines and newspapers need to change so as to bring about a better reviewing atmosphere. The paper also links the reviewing of translations to the general reviewing culture in India, because the former inherits some of the basic flaws of the latter. More importantly than almost anything else the paper proposes, it aims to emphasize the principle that much remains to be improved in the culture of translation itself.
\end{abstract}

Sudhakar Marathe

Translation has always been reviewed, at least in the private domain by the translator himself or herself, because like all other writing naturally translation is self-reflexive and the first reader is always the translator. Most translators also have good friends who 'review' their work constructively and bad friends who praise it merely out of friendship, a disservice if ever there was one. Goodness help the work if the translator himself or herself is negligent of the basic duties and responsibilities of reviewing and reviewers of the work. For then only a mediocre work may result at best. It seemed to me appropriate to state this fact at the outset, since in this paper I am setting out to review reviewing and reviewers, and to criticize them

Translation Today Vol. 5 No. 1 \& 22008 @ CIIL 2008 
with the desire and hope that, even if my arguments do not engender better reviewing of translation, at least we translators may understand the business of reviewing better. After all, if as translators we are not rigorously and punctiliously honest with ourselves, we would have to relinquish our right to criticize reviews and reviewers.

One might have chosen any item from the generous offering of subjects regarding the issue of reviewing translations. Tempting as the somewhat simple choice might have been to select one such items, I have chosen to respond to nine sets of questions, addressing each, at some point asking other questions, and attempting to answer at least some of them. Of necessity my answers or responses to them will be brief. Still, it seemed worth attempting this exercise because I have been translating for thirty years and I am likely to continue to do so; therefore I feel confident that reviewing 'reviewing' will educate me considerably as a translator. That is my hardly concealed personal motive for attempting this task.

Let us consider the first question: 'Why are most reviews of translated works mere summaries of the source texts?' There are a couple of quite respectable answers to this question and a couple that reveal the ugly side of reviewing:

- first, a sort of summary is obviously required if the reader of a review who has, obviously, not read the translation yet, is to know the basic outline of it;

- $\quad$ second, without a summary the points of criticism or praise the reviewer raises with reference to the work would fail to make sense to the reader of the review for lack of context;

- third, reviews are rarely mere reviews. They also frequently perform the function of 'notices', which are meant to bring texts to readers' attention by introducing them.

So there is reasonable justification per se for summarising the plot or outline of a text, although filling column space in this way ought to be anathema. On the other hand, 
- most Indian reviews of all kinds summarize texts (just as most examiners of dissertations summarise them in their reports). Reviews of translation are a mere sub-species of reviews, so one need not imagine that a reviewer has a particular bias against translated texts because he or she summarises their plots;

- besides, summarising fills space in newspaper or magazine columns, a material of extraneous consideration in reviewing. In contrast, I once composed a one sentence review. The book was V. S. Naipaul's Finding the Centre. My review was going to be this: 'It does not.' But who would have printed such a review? And perhaps it would have been too clever by far to be of any use to readers.

Let us look at the second set of questions: 'Why is it that reviews of translated works very rarely mention the name or names of the translators? Even when they do mention them, why do they rarely go beyond saying that the works are good or not good?' One can answer this set of questions reasonably, too, on both sides of the issue but with far less justification for reviewers than in the first case.

- first of all, not mentioning the name of a translator is a cardinal $\sin$ in a translation review. The reviewer apparently just forgets the name while remembering the original author's name. Indeed, in some reviews one hardly finds any awareness that the work in question is a translation;

- one reason for this lapse is that even today in India both readers and reviewers implicitly value the 'original' work and author above a translation. They treat a translation as a mere convenience, purely secondary. Therefore, its 'author', the translator, matters little to them;

- but the matter does not begin or end here. Unfortunately, the publishing world itself tends to devalue a translation vis a vis 
an original. Even after a century and a half of modern translation activity in India, today many publishers refuse to install the translator's name or names on a book's jacket. They appear as unavoidable adjuncts on title pages, but only just. I am afraid reviewers merely 'take a leaf out of the publishers' book,' to pun a little;

- indeed, as the bulk and number of translated work went up during the past half century, so

- much translation turned out to be mediocre or worse, and unsatisfactory on numerous counts, that it has done serious disservice to the trade or craft. On many an occasion it is better that a translator's name remains unknown;

- editors of magazines and newspapers that publish reviews do not hold translation in high regard, and their attitude passes down to reviewers. Unlike some other places, in India reviewers are only very rarely translators themselves, unless they are frustrated translators; so they feel little awareness of the travail of translation; therefore they end up neglecting the translator;

- it is for the same set of reasons that most reviews of translated works actually do not bother to evaluate the success of translation, merely making a judgmental remark or two to the effect that they are good or that they fail to satisfy a 'discerning reader' like the 'esteemed reviewer';

- and finally, editors or review editors rarely seek out respectable or established translators for reviewing, assigning the work to any taker, or worse to an undeserving favourite person. This practice is clearly detrimental to fair and constructive reviewing of translations. A good reviewer (regardless of his or her assessment of a work and bent of mind) knows the 
subject, form and craft of the work reviewed. That is not, alas, usually the case.

Now the third question: 'Does this mean that even when he is reviewing translated works, a reviewer is only reviewing the source text? If so, how is the translation different from a reissue of the source text?'

- well, the first part I have already answered: alas in India source texts, especially when they are already well known, take priority as a mater of implicit principle, or more accurately, cultural hangover;

- this is a deep rooted cultural phenomenon, for in India all things old are venerable. It still pervades the Indian education system in which the teacher's word is supposed to be pramana or Brahma vakyam, even when the teacher is utterly wrong or patently ignorant and incompetent; it still pervades family structures, in which the 'head of the house' is still by and large the oldest male; and in Indian bureaucracy almost without exception the nyaya of 'boss-vakyam pramanam' still operates;

- indeed, in effect, except in saying or suggesting that the translation is of course inferior to the original, this is a view held especially by reviewers who know both the source and target languages and literatures.

Therefore, in fact a translation is often treated like a reissue of the original. Clearly this is an aspect of reviewing that must change. But remember that we often translate a work because it is worthy of special respect and treatment.

The fourth item actually begs the question, because answers to the queries listed in it have been for a very long time part of standard or received views of translation. In principle at least we need no longer ask such questions, but for the fact that reviewers and translators alike 
flout the givens. The basic query is this: 'Doesn't the activity of translating them [source texts] from one language to another have anything to do with the literary, socio-cultural and political climate of the target language and culture?' Of course it does. In fact there is a great deal more to it than even translators normally recognize or acknowledge:

- for instance, despite the popular cry 'Historicize!' they do not recognize that frequently the era or age from which the source text comes is far removed from the era in which translation is being done;

- neither recognizes the fundamental fact that translating written language automatically and invariably implies translating the way the sound of the source language is heard and the way it must be heard in the target language;

- for instance, they do not take into account the register or level and social context of the spoken language in either culture, often coming up with versions that are entirely inappropriate in the target language or do violence to source expressions;

- for instance, far too few translators actually command the source language and its cultural, social, political, economic or historical manifestation or ethos well enough to translate in the first place;

- Recently I was advising someone regarding a translation in which the original (a version of the Ramayana) said that Rama affectionately placed his younger brother Lakshmana 'in his lap'; this was translated into English as 'on his thigh'. The translator attempted to justify the version, saying that he wished to preserve the feel of the original language in it; so, for instance, the cultural value of the old habit of placing even a younger same-sex sibling in one's lap was all lost in the purely physiological and pig headed, poor joke non-equivalent word thigh; 
- let us not forget either that when we select a work to translate, nine times out of ten we do so because it is a very good if not a landmark work in the source language, which is a factor in the valorisation of the source text too;

- and finally in this connection, of course at least in theory, a translation hopes to bring something to the 'target language culture', a social input, a literary input, a model of personality or behaviour or ideology, a phenomenon considered historically appropriate for introduction in the given conditions in the target society.

One ought to list all the types of detail which a translator must notice, understand, appreciate, and culturally and historically locate in the source language context the text in question before he or she attempts to do the same in the target language. Therefore, I exhort all fellow translators never to allow reviewers' failure to take such matters into account to hide the far more damning fact that most translators also fail to do so.

A different kind of question confronts us in the fifth subject: 'What kind of changes (if any) does the translated text seek to bring about in the target language culture by an act of translation?' This question concerns motivations for translating. And if at times bringing about a change in the target language and culture happens to be a motive, however doubtful it may be, it must not be presumed in every case. Let us begin with motives at the farthest end from this one:

- today the commonest case of translation involves being asked or commissioned to translate something, not choosing to translate something; in such a situation initially one has almost no motive except to carry out the assigned work;

- not infrequently, again, a text is translated because it is part of a larger scheme, and either one is asked to translate it or one translates it because 'it comes with the territory'; and while 
one may have an 'agenda' for the whole scheme or project, one may not necessarily have one for the specific text in question;

- at times one translates a text from another language into one's mother language or proposes that it be translated into another language because one likes it and believes that others might like it too, therefore it ought to be made available to them;

- on occasion a text is seen as historically important and therefore worth translating; novels or poetry that influenced trends in literature in one's own language are cases in point. But here too one wishes not so much to bring about a change but to make a source of influence available to readers. An obvious example is T. S. Eliot's Waste Land and its translations in Marathi or Malayalam;

- on other occasions one translates a work because it happens to be important to someone else, as is the case with a technical document, and once again the motivation is to make it available to 'readers' in the target language. Here is an example from my own work: some years back I translated the Hindu Wedding Ceremony and Ritual from Sanskrit into English (with a great deal of help from a Marathi paraphrase of course) for a friend of my daughter who was marrying a Catholic and wanted the groom's family to be able to understand the language and rituals of the wedding;

- the second instance was a translation into Marathi of two T. S. Eliot essays titled 'Studies in Contemporary Criticism' I and II, because in this case, in my opinion Marathi criticism needed an injection of objectivity, which was the main focus of the essays. 
Let us now consider the question concerning motivation for translation:

- there are texts which evince some political or other approach or theory that one wishes others to know, either from another language into one's own or vice versa, the idea being to affect thinking on an important subject;

- in a similar category come historical or cultural documents (literary or otherwise) that contain viewpoints or facts or both that are not 'received' but that would change the complexion and interpretation of some important phenomenon or event and one wishes to translate them to effect such a change;

- next comes translation of documents that present models of style, construction, form or subject matter that do not exist in one's language or vice versa, and one wishes to translate them so as to import or export them to affect the literature in the target language;

- then comes translation of documents of which one expects to establish the greatness or level of achievement in one's culture and literature, such as (auto)-biographies of important figures or literary 'masterpieces', with the express desire to alter the evaluation of the literature and culture of the source language in the eyes of the target readership;

- and there is at least one more motivated type of translation, that of documents to fill gaps in knowledge or history, documents of literary, political, historical or cultural kind translated in either direction.

It ought to become clear from this variety that if a reviewer does not know the motivation for a translated work, a review is at least likely to remain partial, or actually become unfair in assessment of the work. Take for instance a translated work of a Dalit writer; if the reviewer fails to take into consideration the enormously 
complicated and long linguistic-cultural-historical-political background of Dalit life in India, he or she is unlikely to contextualize the work or make a genuinely acceptable assessment of it. It is equally certain that if a reviewer remains ignorant of the context of the source work and the social and other kinds of context into which the target work would be received, he or she would be unable to assess the technique and intended effect of the translated work. Consequently, again, his or her review must remain partial or become unfair in assessment of the impact of such a work.

I believe that I have already implied in the foregoing discussion the answer to the next question, the changes that a translator may wish to bring about in the target language and culture: political and cultural changes, reassessment of all kinds of social or literary phenomena, changes in historical perspective either in social or in literary terms, changes in attitude among the readership that might result in such changes, changes even in the laws of a land where translated works reveal lacunae in them. Before India's independence, for instance, it would have been genuinely useful and effective to have translated Jonathan Swift's “A Modest Proposal” into Indian languages.

I must say, however, that to expect a reviewer to take all such motivation into account, someone somewhere, including the translator, must at least hint at the possibility. The prefatory statement to a work, an introduction, a note sent to reviewers for edification, some such device must make sure that the reviewer is forewarned in this regard. I am not sure that translators always do this or that publishers of translations or organs in which reviews appear are bothered to include such explanations in their publications. All the same an even more crucial factor rears its head here: whatever the motivation of the translator, it is a fundamental question whether the translation itself has succeeded in achieving either shape or style or tenor or language that might reflect the contents and tone of the source work that may affect target readership or society. A translator who fails in this regard can hardly blame a reviewer for doing so. Therefore, at this point I would be less than honest if I did not reflect my personal assessment 
of the bulk of Indian translation activity either at least into Marathi or from Marathi into English: by and large its results are of inferior quality, so it fails miserably to make any impact at all, let alone making a significant political, cultural or social impact. For this failure it would be unfair to blame either reviewers or reviewing.

The next, the sixth question puzzles me somewhat. For all its potential power, translation is hardly known for affecting the source language, work or culture much. It is of course possible that a translator may wish to translate a work in such a way as to bring about a change in the perception and evaluation of the source text in the target language. A significant translation can also reveal qualities or aspects of a source text that readers in the source language or in translation in the target language may have missed altogether. If such is the case and such the motivation, either in favour of or against over-valuation or wrong valuation of a work, one can easily understand the translator's wish or desire. I cannot see this happening too frequently, however. It has not happened to Sharatchandra or Bankimchandra in Marathi, for instance, or to any English or European writer that I am aware of. A text worth re-translating because the first translation was altogether wrong may bring about a reassessment in the minds of the target readership or even contemporary source society. A case in point would have been available if Vyankatesh Madgulkar's fabulous little Marathi novel Bangarwadi had been re-translated into English. But the original publishers chose, against the wishes of the writer, to republish after a gap of three decades the first terrible stilted translation. Yet even if a new translation had appeared, it could not have retrospectively affected in any way the Marathi society of half a century ago. Alas, not even translation has the power to modify history retrospectively.

On the other hand, the second part of the question considered goes way beyond this issue, as it concerns the possibility of effecting changes in the 'climate' of a source text. It seems to me impossible for a translation to bring about a change in the social, political, economic, cultural or ideological climate in which in the source culture a source text was originally published. It could only happen if the 
translation is virtually instantaneous. Yet even then how much effect on the source society a translation in a language the source readership does not know can create is a serious question, indeed.

The next, seventh, question directs our attention to 'whether it is necessary for the review to reflect on the quality of the translation,' and whether there is something that may be called the style of the translation that needs to be analysed. Of course a review must consider the quality of a translation. What else can we consider the central or essential task of a review? But if the question implies that reviews by and large fail to satisfy this need, one would have to agree without hesitation. Unfortunately, Indian reviews rarely analyse translations qualitatively. The reasons for this failure are, again, many:

- reviewers who do not practise or understand translation or are incapable of evaluating its quality have no business reviewing it;

- reviewers who dare not approach the subject of quality at all either for fear of exposing their inability or the poverty of a translation have no business reviewing it;

- other questions do arise, especially in newspaper reviewing: for instance, whether it is worthwhile reviewing a failed translation, or how such a task may be managed, and so on;

- besides, in a country in which honest and relentless assessment of any work, whether literary or not, is extremely uncommon, one can hardly expect reviewers of translation to become exceptions and manifest those qualities;

- purely personally, I also believe that the bulk of translated work in India (at least into Marathi or from Marathi into English) would be lambasted by reviewers if they were to fulfil the requirement of supplying honest assessment of the quality of translation; whether the situation is comparable in other languages I can guess at but cannot vouch for; 
- as for the style of a translated work, although my previous remark applies in general, of course any worthwhile translation does have its own style and it ought to be considered, commented upon and evaluated by a review: but reviewers rarely do so mainly because detailed analysis of texts has never been the strong point of Indian criticism in general. So we may be unwise to expect such criticism only from translation reviews.

Such analysis ought to happen, of course. But that requires a spread or expansiveness in a review that neither magazines nor newspapers allow a reviewer. As with reference to most of what I have said before, even this issue brings to the fore the necessity of a new culture of translation reviewing, but I shall have more if brief questions to raise on that subject later.

The eighth question is much more to the point, given our main issue: 'Is it necessary for the reviewer to know both the source and target languages? [Or] Is it enough for her / him to be familiar with the source and target language cultures?'

- this too begs the question, regarding whether a reviewer can know both source and target cultures without knowing either language well and without being well read in both;

- it does not seem to me at all possible for a reviewer to know both cultures well enough to qualify for reviewing while remaining outside the two languages: even purely contemporary culture is so complex that to know both well enough without knowing or reading the languages in question seems unlikely enough; but since any culture has a long history, it is impossible to obtain knowledge about it without extensive and sensitive reading in its language, because it is in language that culture is mainly recorded and preserved. That is why I wonder why such a question arose at all; 
- all the same, the question whether a reviewer ought to know both the source and target languages of a translated text is a valid one; and I for one would not hesitate to say that, normally, no one should review a translation without knowing both languages and cultures well enough to read the translation meaningfully in the first place;

- in fact, going much further, I would say that like a translator a reviewer must know both languages and cultures very well, even if it is only very well. There is no upper limit on such knowledge, of course, but the lowest expectation would still require a reviewer to be well versed in both, for why else would he or she wish to review at all?

But, as before, let me also point out that whatever standards we set for the reviewer must be at least equalled if not topped by the translator. Or we can in turn ask why he or she should translate at all.

The final or ninth set of questions concerns publication of reviews. Whether we have here asked at least all the basic questions or not, clearly this is an important subject for us: 'How important is it for the source text, the writer of the source text and reviewer that a review appears in a regional / national / international publication? Also, is there a difference between a review of a translated text appearing in the source language and a review appearing in the target language?'

- first, surely, the question would concern both local and national or international publication.

- next, whether it should be local is hardly open to question: at least in the target language reviews must appear in the local organs;

- whether reviews also appear in the source language is a matter of concern in the source community for local literature and its transmission to other language communities and cultures; but 
ideally reviews of translations ought to appear in the source language as well: in fact, they rarely do;

- whether reviews appear in the national press is a question that would be redundant if a nation had only one or one dominant language, as would be the case of English in New Zealand, for instance;

- the question of publication of reviews on the national level is a natural one in India, however, for we are now so used to considering two stages for Indian languages, local and national. Given this, for the sake of everyone concerned, reviews of at least significant works of translation ought to appear on the national stage as well. Whether there is a place on earth where reviews of regional language translations can legitimately appear nationally for the hundreds of works annually translated is another question;

- but whether nationally or internationally, the question would be asked whether a work in either language is significant enough for reviewing. Who can answer that question is a moot point, but it will be asked and by and large it will be answered in the negative for most translated works. Nor does it seem possible for a translator to do much more than hope for favourable reviews on the larger stage;

- and surely there is a difference between same-language reviews and other-language reviews: indeed, same-language reviews would differ qualitatively from others because invariably their major issue and focus would be success in rendering the source language; personally, I have always felt that almost no same-language reader or reviewer is likely to be satisfied with a translation, because he or she knows the original text too well to miss the inevitable lacunae in the translation; also let us say that it is almost a 'professional 
hazard' for a reviewer to feel that he or she would have translated the work better;

- on the other hand a review entirely based on knowledge of source language and culture too must lack the advantage of knowing how effective the translation happens to be; such a review may very well be able to opine on the success of a text in the source language as though the text being reviewed were written in that language, but it cannot evaluate the work qua translation.

Now I believe one might spend just a little time on a few important issues I have hinted at several times: the nature and responsibility of reviewing translations; the qualifications of a reviewer; the likelihood of a translation being reviewed at all; the 'standards' or criteria that reviewers may or ought to use; the responsibility of organs such as newspapers and journals regarding reviews; the possibility of initiating and sustaining specialist journals or columns for translation reviews; the gap between being reviewed and not reviewed; being filled by a culture of notices in newspapers or journals; and a culture of reading and reviewing translations that is serious enough for it to make a difference to the general culture of our society.

There is little doubt that in India there is inadequate reviewing of translations; that such reviewing as does occur is often beside the point or fails to address core issues; that perhaps we require a set of journals or significant portions of existing journals exclusively devoted to translation reviewing, for which purpose publishers and editors of newspapers and magazines need to be educated concerning the importance of translation; that highly qualified as well as sensitive reviewers alone ought to review translations; but, at the same time, translators ought to be honest and open-minded enough to confront criticism and valuations lower than or different from their own; just as translators ought to take their own work seriously enough to apply equally stringent standards to their work in every aspect of translation. 
For one thing cannot be denied-no translation will be good merely because the translator considers or wishes it to be good. A work is meant for readership. And while we hope that reviewers, editors and publishers as representatives of that readership will work better and with better standards and rigorousness than at present, we could hardly make such a demand of them without making exactly the same demand of ourselves as translators! I must say that I for one am as pessimistic about the latter happening as about the former. Let us set our own house in order at the same time as we demand improvement in the House of Reviews. Ultimately, what happens in reviews of translation forms part of and reflects the general literate culture of a society. And obviously on many fronts we all have a very long way to go to achieve a vital culture of reception of translation. We need, I believe, to collaborate in this regard with publishers, editors and reviewers, rather than assume a posture of confrontation with them. 